\title{
Phase 2 clinical trial of PBI-4050 in patients with idiopathic pulmonary fibrosis
}

\author{
Nasreen Khalil ${ }^{1}$, Helene Manganas ${ }^{2}$, Christopher J. Ryerson ${ }^{3}$, Shane Shapera ${ }^{4}$, \\ Andre M. Cantin ${ }^{5}$, Paul Hernandez ${ }^{6}$, Eric E. Turcotte ${ }^{7}$, Joseph M. Parker ${ }^{8}$, \\ John E. Moran ${ }^{8}$, Gary R. Albert ${ }^{8}$, Renata Sawtell ${ }^{8}$, Aline Hagerimana ${ }^{8}$, \\ Pierre Laurin ${ }^{8}$, Lyne Gagnon ${ }^{8}$, Frank Cesari ${ }^{8}$ and Martin Kolb $\mathbb{1}^{9}$
}

Affiliations: 'Vancouver General Hospital - The Lung Centre, Vancouver, BC, Canada. ${ }^{2}$ Centre Hospitalier de l'Université de Montréal, Montréal, QC, Canada. ${ }^{3}$ Dept of Medicine, University of British Columbia and Centre for Heart Lung Innovation, St. Paul's Hospital, Vancouver, BC, Canada. ${ }^{4}$ University Health Network, University of Toronto, Toronto, ON, Canada. ${ }^{5}$ Centre de Recherche Clinique du Centre Hospitalier Universitaire de Sherbrooke (CHUS-CRC), Sherbrooke, QC, Canada. ${ }^{6}$ Queen Elizabeth II Health Sciences Centre, Halifax, NS, Canada. ${ }^{7}$ Centre d'Imagerie Moléculaire de Sherbrooke, Université de Sherbrooke, Sherbrooke, QC, Canada. ${ }^{8}$ Prometic Life Sciences Inc., Laval, QC, Canada. ${ }^{9}$ Firestone Institute for Respiratory Health, St. Joseph's Healthcare, Hamilton, ON, Canada.

Correspondence: Martin Kolb, Firestone Institute for Respiratory Health, St. Joseph's Healthcare, 50 Charlton Avenue East, T2120, Hamilton, ON, L8N 4A6, Canada. E-mail: kolbmamcmaster.ca

@ERSpublications

PBI-4050 alone and in combination with nintedanib demonstrated no safety concerns and showed encouraging results for lung function in IPF patients http://ow.ly/olQD30myD0E

Cite this article as: Khalil N, Manganas $\mathrm{H}$, Ryerson CJ, et al. Phase 2 clinical trial of PBI-4050 in patients with idiopathic pulmonary fibrosis. Eur Respir J 2019; 53: 1800663 [https://doi.org/10.1183/ 13993003.00663-2018].

ABSTRACT PBI-4050 is a novel orally active small-molecule compound with demonstrated anti-fibrotic activity in several models of fibrosis, including lung fibrosis. We present results from our first clinical study of PBI-4050 in patients with idiopathic pulmonary fibrosis (IPF).

This 12-week open-label study explored the safety, efficacy and pharmacokinetics of daily oral doses of $800 \mathrm{mg}$ PBI-4050 alone and in combination with nintedanib or pirfenidone in patients with predominantly mild or moderate IPF. Nine patients received PBI-4050 alone, 16 patients received PBI-4050 with nintedanib and 16 patients received PBI-4050 with pirfenidone.

PBI-4050 alone or in combination with nintedanib or pirfenidone was well tolerated. Pharmacokinetic profiles for PBI-4050 were similar in the PBI-4050 alone and PBI-4050+nintedanib groups but reduced in the PBI-4050+pirfenidone group, suggesting a drug-drug interaction. There were no significant changes in forced vital capacity (FVC), either in \% predicted or $\mathrm{mL}$, from baseline to week 12 for PBI-4050 alone or PBI-4050+nintedanib. In contrast, a statistically significant reduction $(\mathrm{p}<0.024)$ in FVC $\%$ pred was seen for PBI-4050+pirfenidone after 12 weeks.

There were no safety concerns with PBI-4050 alone or in combination with nintedanib or pirfenidone in IPF patients. The stability of FVC between baseline and week 12 looked encouraging for PBI-4050 alone and in combination with nintedanib.

This study is registered as a clinical trial (NCT02538536). The study protocol and individual participant data that underlie the results reported in this article, after de-identification (text, tables, figures and appendices), will be made available to researchers who provide a methodologically sound proposal directed to the Chief Medical Officer at Prometic.com. Data will be made available 9-12 months following article publication, until 36 months following article publication. To gain access, data requestors will need to sign a data access agreement. Data will be available at a third-party website.

Received: April 062018 | Accepted after revision: Oct 282018

Copyright $\odot$ ERS 2019. This article is open access and distributed under the terms of the Creative Commons Attribution Non-Commercial Licence 4.0. 


\section{Introduction}

Idiopathic pulmonary fibrosis (IPF) is a chronic, irreversible, progressive and usually fatal lung disease of unknown cause $[1,2]$. It is characterised by scarring of the lung parenchyma, progressive loss of lung function, dyspnoea and cough, eventually leading to respiratory failure [1]. IPF occurs primarily in older adults, with a median age at diagnosis of 66 years. Across Europe and North America, the incidence of IPF ranges from three to nine cases per 100000 person-years [3] and appears to be rising. The prevalence has been reported as high as 45-199 per 100000 in individuals 60-79 years old [4]. Median survival is $3-4$ years after diagnosis $[1,5,6]$.

Current clinical practice guidelines recommend the use of pirfenidone or nintedanib for the treatment of IPF $[1,7]$. However, both drugs have limitations in terms of efficacy and tolerability. Therefore, additional therapies are needed to treat this progressive and deadly disease [8].

Although inflammation may play a role in the initial injury to the lung in IPF, the primary process is an epithelial-dependent, fibroblast-activated progressive fibrotic process [2]. The trigger for IPF is thought to be the inability of the alveolar type II cells to self-renew and repair, leading to the release of fibrotic factors $[2,9-11]$. This injury results in fibroblast recruitment, proliferation and differentiation into myofibroblasts, which lay down collagen and extracellular matrix proteins, resulting in scar formation $[10,12,13]$.

PBI-4050, 3-pentylbenzeneacetic acid sodium salt, is a first-in-class, orally active, low molecular weight compound in clinical development for the treatment of fibrotic diseases. It is a synthetic analogue of a medium-chain fatty acid that displays agonist and antagonist ligand affinity towards the G-protein coupled receptors GPR40 and GPR84, respectively, leading to the reduction or reversal of fibrosis by regulating macrophages, fibroblasts/myofibroblasts and epithelial cells [14]. By binding GPR40 and GPR84, PBI-4050 reduces fibrosis via the regulation of multiple anti-fibrotic pathways implicated in the pathogenesis of IPF [14]. PBI-4050 inhibits the differentiation of fibroblasts to myofibroblasts, as demonstrated by abrogation of $\alpha$-smooth muscle actin expression in fibroblasts and subsequent accumulation of extracellular matrix protein deposition and fibrosis. PBI-4050 reduces the expression of both pro-inflammatory markers (monocyte chemoattractant protein-1, interleukin (IL)-8 and IL-6) and pro-fibrotic markers (connective tissue growth factor and IL-6). PBI-4050 also significantly attenuates fibrosis in kidney, liver, lung, heart, pancreas and skin fibrosis models, including the murine model of bleomycin-induced lung fibrosis [14]. In the latter model, PBI-4050 resulted in a $47 \%$ reduction of histological lesions depicted as disrupted lung architecture, thickness of alveolar wall and fibrosis [14]. These findings suggest that PBI-4050 may be clinically effective in fibrotic diseases, including IPF.

A series of phase 2 exploratory studies of PBI-4050 have been completed or are ongoing in patients with fibrotic diseases, including IPF, type 2 diabetes with metabolic syndrome and Alström syndrome. Herein, we present data from a phase 2 open-label study evaluating the safety, efficacy and pharmacokinetics (PK) of PBI-4050 in patients with IPF receiving nintedanib, pirfenidone or neither.

\section{Methods \\ Study design}

This was a 12-week phase 2 single-arm open-label study (NCT02538536) in adults with IPF conducted at six sites across Canada. Its primary purpose was to evaluate the safety and tolerability of PBI-4050 in this patient population. This study also explored the effect of PBI-4050 on pulmonary function. A subset of patients volunteered to take part in a PK sub-study to evaluate the PK profile of PBI-4050. An open-label design was chosen owing to the exploratory nature of the study.

The protocol was reviewed and approved by the institutional review board of each participating centre and the study was conducted in accordance with the Declaration of Helsinki and good clinical practice guidelines. All patients provided written informed consent prior to entering the study.

\section{Study population}

Patients were eligible if they were $\geqslant 40$ years old and had been diagnosed with IPF as determined by the investigators as per guidelines from the American Thoracic Society (ATS), European Respiratory Society (ERS), Japanese Respiratory Society (JRS) and Latin American Thoracic Association (ALAT) [1]. High-resolution computed tomography (HRCT) scans of the thorax were performed at screening or obtained within 12 months prior to the start of study drug treatment. HRCT scans performed at the site followed a standardised volumetric acquisition protocol that provided multi-planar reformations (coronal and sagittal) of the entire lung, improving the evaluation of abnormality distribution and the extent of disease. Although all scans were sent to a central reader (Centre Hospitalier Universitaire de Sherbrooke, Quebec, Canada) for review, confirmation of IPF by a central reader was not factored into patient eligibility. There were no eligibility criteria based on pulmonary function tests. HRCT was also used to assess any intercurrent infection. 
Key exclusion criteria included a known diagnosis of a respiratory disorder other than IPF; extent of emphysema greater than the extent of fibrotic changes (honeycombing and reticular changes) on HRCT; and being an active smoker within 3 months prior to screening, because smoking may worsen the progression of IPF and reduce the efficacy of background anti-fibrotic therapy [15-17].

\section{Treatment}

All patients received PBI-4050 at a dose of $800 \mathrm{mg}$ once daily with a planned treatment duration of 12 weeks, administered orally as $4 \times 200 \mathrm{mg}$ gel capsules $\geqslant 1 \mathrm{~h}$ before or $>2 \mathrm{~h}$ after a meal. This dose was based on the PK profile of PBI-4050 from phase 1 studies [18] that provided the same area under the concentration-time curve as the effective dose of PBI-4050 $\left(200 \mathrm{mg} \cdot \mathrm{kg}^{-1}\right)$ in the murine model of bleomycin-induced lung fibrosis [14]. This dose was also well tolerated in healthy volunteers and patients with stable renal impairment [18]. Nintedanib and pirfenidone were allowed during the study, as was the use of corticosteroids. Azathioprine and $\mathrm{N}$-acetylcysteine were prohibited based on ATS/ERS/JRS/ALAT recommendations against these agents for the majority of IPF patients [1].

\section{Analysis}

The primary objective of this study was to examine the safety and tolerability of PBI-4050, with the following endpoints: patient-reported adverse events, clinical laboratory tests, vital signs, ECGs and physical examinations. Adverse events, defined as either new events emerging after study drug administration or previous events that increased in severity after study drug administration, were monitored throughout the treatment period until the follow-up visit (defined as 28 days after the last dose of study drug). Clinical laboratory tests and vital signs were measured at weeks 1, 2, 6 and 12 and at follow-up; physical examinations were performed and ECGs recorded at weeks 1 and 12 and at follow-up. All safety endpoints were analysed descriptively.

The secondary objective was the assessment of clinical efficacy, which was measured by mean changes from baseline in pulmonary function tests (forced vital capacity (FVC) and haemoglobin-corrected diffusing capacity of the lung for carbon monoxide (DLCO)). FVC was measured at weeks 1, 2, 6 and 12 and DLCO was measured at weeks 1 and 12; both FVC and DLCO were measured by local laboratories using standard procedures, in accordance with ATS/ERS standards [19, 20]. All efficacy measurements were analysed descriptively.

The PK profile of PBI-4050 was also a secondary objective and comprised individual and mean plasma concentration versus time curves at week 12 as part of a separate sub-study. For this sub-study, blood samples were collected under fasting conditions at the following time points: pre-dose and $0.5,1,2,3,4$ and 5-6 h post-dose. Descriptive statistics were used to describe the PK of PBI-4050. The PK profiles of pirfenidone and nintedanib were not examined in this study.

This study was exploratory in nature with a primary objective of testing the safety of PBI-4050; therefore, no power analysis or between-treatment group comparison was performed.

This study was designed as a single-arm trial, with all patients analysed as a single group for all primary analyses. A post hoc analysis was also conducted with patients grouped according to their background IPF pharmacotherapy (PBI-4050 alone, PBI-4050+nintedanib, PBI-4050+pirfenidone). As part of this analysis, exploratory hypothesis testing was performed within each treatment group to assess differences from baseline to week 12 in FVC and DLCO. Exploratory p-values (t-test) and 95\% CIs are provided. There were minimal missing data and imputation was not needed. Multiplicity testing was not applied across the efficacy endpoints.

\section{Results}

Between October 2015 and January 2017, 52 patients were screened and 41 patients were enrolled (figure 1). All but one patient completed the study as planned (figure 1). The remaining patient (PBI-4050 +pirfenidone) withdrew early from the study owing to an IPF exacerbation that occurred within 2 weeks of starting the study drug. Nine patients received PBI-4050 with no background IPF pharmacotherapy (PBI-4050 alone), 16 patients received PBI-4050 with nintedanib (PBI-4050+nintedanib) and 16 patients received PBI-4050 with pirfenidone (PBI-4050+pirfenidone). Nine patients (two, four and three patients, respectively) were included in the PK sub-study.

\section{Baseline patient demographics and disease characteristics}

Demographic and baseline characteristic data are summarised in table 1. In general, the overall study population characteristics were consistent with an underlying disease of IPF, with all but two patients (95\%) having a definite usual interstitial pneumonia (UIP) pattern on HRCT (the remaining patients had a possible UIP pattern). Patients ranged in age from 46 to 83 years, and most patients were male and 


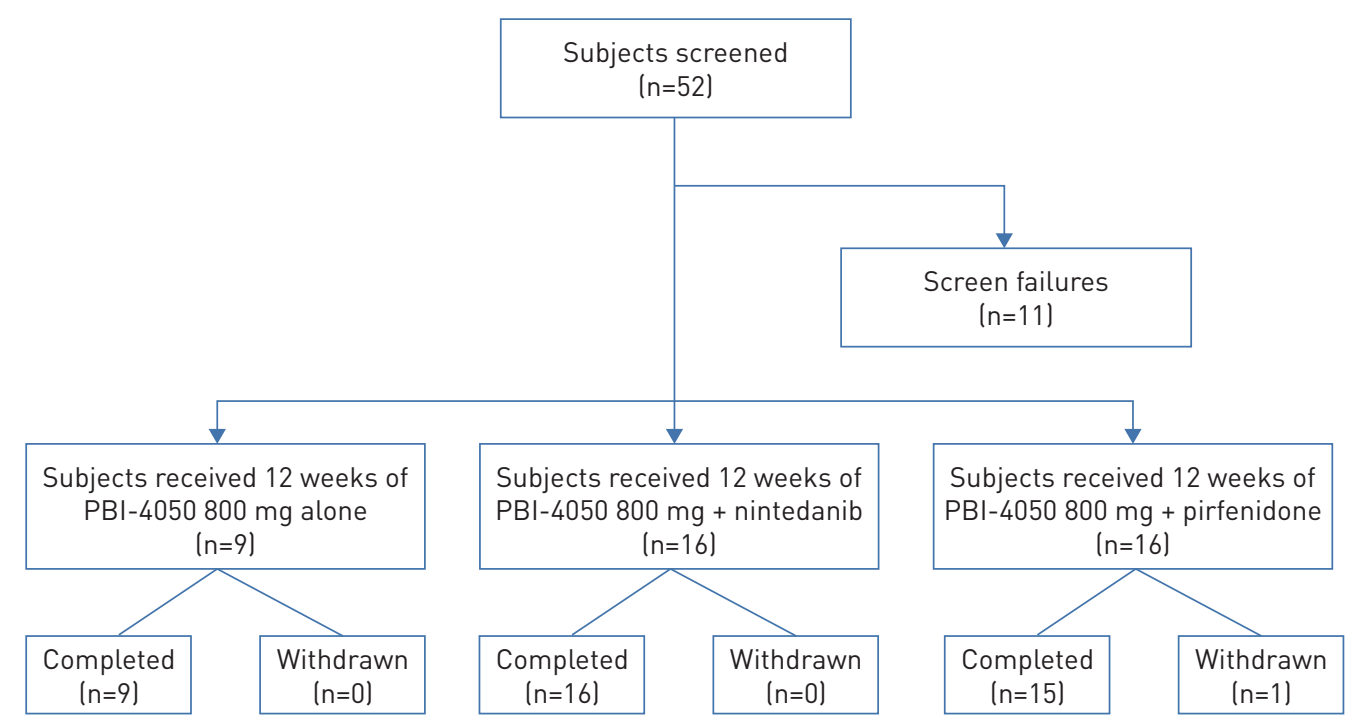

FIGURE 1 Patient disposition.

Caucasian. Other baseline characteristics were more variable across the treatment groups. Mean/median weight was higher in the PBI-4050+pirfenidone group than in the other two treatment groups, but these differences did not reach statistical significance and appeared to be associated with a few outliers. Patients in the PBI-4050+pirfenidone group were generally more recently diagnosed and had a higher proportion of moderate or severe disease than the other two treatment groups. Mean/median FVC \% predicted was similar between the two combination groups but highest in the PBI-4050 alone group.

\section{Safety and tolerability}

All patients who received at least one dose of PBI-4050 were included in the safety analysis. Most patients $(\sim 83 \%)$ had at least one adverse event as summarised in table 2 . The majority of events were mild or moderate in severity and not serious. Three patients experienced severe adverse events, which included diarrhoea (PBI-4050+nintedanib), dyspnoea and IPF exacerbation (PBI-4050+pirfenidone) and gastrointestinal disorder (PBI-4050+pirfenidone); none of these events were related to PBI-4050. No patients died, and one patient in the PBI-4050+nintedanib group had a serious adverse event (pneumonia) that was not related to PBI-4050. In addition, one patient in the PBI-4050+pirfenidone group discontinued treatment owing to an adverse event (IPF exacerbation) related to their underlying disease. The most frequent adverse events were diarrhoea, nausea and headache. There were no clinically significant changes in clinical laboratory test results, vital signs, physical examination findings or ECGs.

\section{Pharmacokinetics}

The average PK profiles for the PBI-4050 alone and PBI-4050+nintedanib groups were similar (figure 2). The PK profile of the PBI-4050+pirfenidone group, however, demonstrated a reduced absorption rate or lower maximum observed plasma concentration and a faster metabolism (shorter half-life) compared with the other treatment groups, suggesting a drug-drug interaction between PBI-4050 and pirfenidone.

\section{Efficacy}

Pulmonary function test results are shown in table 3. Only patients who received all 12 weeks of treatment were included in the efficacy analysis. No statistically significant changes were observed in mean FVC (\% pred or $\mathrm{mL}$ ) from baseline to week 12 in the PBI-4050+nintedanib group $(0.06 \%, \mathrm{p}=0.9513 ; 1.87 \mathrm{~mL}$, $\mathrm{p}=0.9539)$ or in the PBI-4050 alone group $(-1.11 \%, \mathrm{p}=0.4759 ;-12.2 \mathrm{~mL}, \mathrm{p}=0.7959)$ (table 3$)$. In the PBI-4050+pirfenidone group, there was a statistically significant mean decrease in FVC (\% pred and $\mathrm{mL}$ ) from baseline to week $12(-2.69 \%, \mathrm{p}=0.0240 ;-102 \mathrm{~mL}, \mathrm{p}=0.0124)$. This was not impacted by the disparity in baseline weight, because there was no correlation between FVC reduction (\% pred and $\mathrm{mL}$ ) and individual weight in this group: $62 \mathrm{~kg}: 7 \%$ and $220 \mathrm{~mL} ; 69 \mathrm{~kg}: 10 \%$ and $280 \mathrm{~mL} ; 78 \mathrm{~kg}: 6 \%$ and $250 \mathrm{~mL}$; $84 \mathrm{~kg}: 6 \%$ and $250 \mathrm{~mL} ; 88 \mathrm{~kg}: 5 \%$ and $200 \mathrm{~mL} ; 113 \mathrm{~kg}: 5 \%$ and $220 \mathrm{~mL}$.

The mean changes in mean DLCO \% pred from baseline to week 12 were not statistically significant in any treatment groups (PBI-4050 alone: $-4.00 \%, \mathrm{p}=0.1427$; PBI-4050+nintedanib: $-1.50 \%, \mathrm{p}=0.1073$; PBI-4050 +pirfenidone: $-2.54 \%, \mathrm{p}=0.1390$; table 3 and figure 3 ). 
TABLE 1 Baseline characteristics of the study population

Characteristic

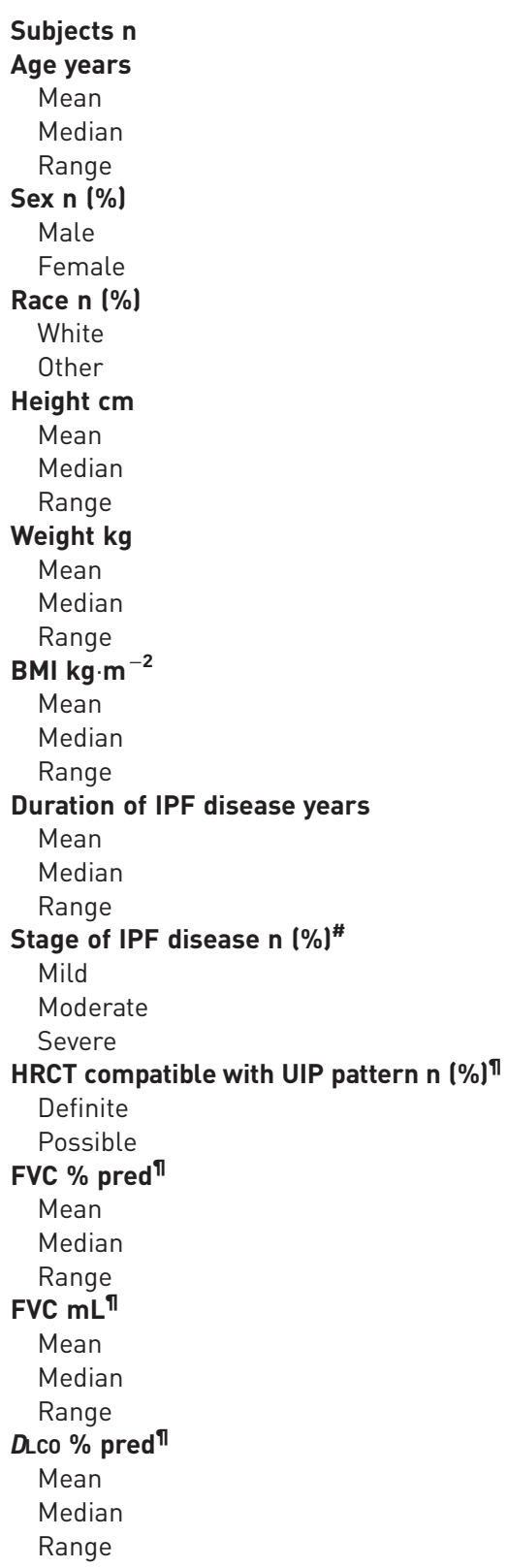

PBI-4050 alone $\mathrm{PBI}-4050+$ nintedanib

PBI-4050+pirfenidone

\begin{tabular}{|c|c|c|}
\hline 9 & 16 & 16 \\
\hline 71.6 & 69.4 & 66.1 \\
\hline 69.0 & 72.0 & 65.5 \\
\hline $65.0-84.0$ & $46.0-83.0$ & $58.0-80.0$ \\
\hline $6(66.7)$ & 12 (75.0) & 13 (81.3) \\
\hline 3 (33.3) & $4(25.0)$ & 3 (18.8) \\
\hline 9 (100.0) & 15 (93.8) & $16(100.0)$ \\
\hline 0 & $1(6.3)$ & 0 \\
\hline 168.0 & 170.8 & 171.5 \\
\hline 172.0 & 171.0 & 170.5 \\
\hline 151.0-178.0 & $157.0-186.0$ & $153.0-193.0$ \\
\hline 76.8 & 76.3 & 89.0 \\
\hline 78.8 & 74.5 & 86.2 \\
\hline $61.5-87.0$ & $57.5-97.0$ & $62.1-130.4$ \\
\hline 27.3 & 26.1 & 30.4 \\
\hline 26.6 & 25.7 & 28.4 \\
\hline $23.4-35.1$ & $23.3-31.0$ & $23.8-55.7$ \\
\hline 2.80 & 1.84 & 1.24 \\
\hline 2.1 & 1.7 & 0.9 \\
\hline $0.8-5.4$ & $0.0-5.9$ & $0.1-5.7$ \\
\hline $5(55.6)$ & $9(56.3)$ & $6(37.5)$ \\
\hline $4(44.4)$ & $6(37.5)$ & $8(50.0)$ \\
\hline 0 & $1(6.3)$ & $2(12.5)$ \\
\hline $9(100.0)$ & 15 (93.8) & 14 (93.3) \\
\hline 0 & $1(6.3)$ & $1(6.7)$ \\
\hline 83.1 & 71.3 & 70.8 \\
\hline 79.0 & 69.0 & 68.0 \\
\hline $58.0-109.0$ & 45.0-107.0 & $45.0-100.0$ \\
\hline 2884 & 2761 & 2849 \\
\hline 2650 & 2735 & 2930 \\
\hline $1750-4380$ & $1290-3480$ & $1580-4280$ \\
\hline 45.2 & 50.8 & 49.1 \\
\hline 47.0 & 53.0 & 45.0 \\
\hline $27.0-64.0$ & $24.0-70.0$ & $23.0-83.0$ \\
\hline
\end{tabular}

BMI: body mass index; IPF: idiopathic pulmonary fibrosis; HRCT: high-resolution computed tomography; UIP: usual interstitial pnuemonia; FVC: forced vital capacity; DLCO: diffusing capacity of the lung for carbon monoxide. ": stage as determined by the investigator; " : data based on efficacy population.

\section{Discussion}

Positive results from a murine model of bleomycin-induced lung fibrosis [14] provided the impetus for the first clinical study of PBI-4050 in patients with IPF. The aim of this study was to evaluate the safety and tolerability of PBI-4050 after 12 weeks of treatment and to assess its PK and efficacy in this patient population. This study was exploratory in nature and not pivotal. An open-label design was chosen to maximise exposure to PBI-4050 and concomitant therapy with nintedanib or pirfenidone was allowed.

Daily oral administration of PBI-4050 at a dose of $800 \mathrm{mg}^{- \text {day }^{-1}}$ over a 12 -week period was well tolerated in IPF patients when given alone or in combination with nintedanib or pirfenidone. No patients died, and 


\begin{tabular}{lccc} 
TABLE 2 Overall summary of adverse events & & \\
Parameter & PBI-4050 alone & PBI-4050+nintedanib & PBI-4050+pirfenidone \\
\hline Total patients & 9 & 16 & 16 \\
Patients with at least one AE & $8(88.9)$ & $12(75.0)$ & $14(87.5)$ \\
Patients with at least one severe AE & 0 & $1(6.3)$ & $2(12.5)$ \\
Patients with at least one serious AE & 0 & $1(6.3)$ & 0 \\
Deaths & 0 & 0 & 0 \\
AEs leading to permanent & 0 & 0 & $1(6.3)$ \\
discontinuation of study drug & & & \\
AEs in >2 patients, by preferred term $\#$ & $3(33.3)$ & $7(43.8)$ & $6(37.5)$ \\
Diarrhoea & 0 & 0 & $4(25.0)$ \\
Nausea & $2(22.2)$ & $1(6.3)$ & $1(6.3)$ \\
Headache & 0 & $1(6.3)$ & $2(12.5)$ \\
Fatigue & $1(11.1)$ & 0 & $2(12.5)$ \\
Non-cardiac chest pain & 0 & 0 & $3(18.8)$ \\
Cough & 0 & 0 & $3(18.8)$ \\
Dyspnoea & & &
\end{tabular}

Data are presented as $\mathrm{n}$ or $\mathrm{n}(\%)$. Patients can be counted in more than one category. AE: adverse event.

\#: Medical Dictionary for Regulatory Activities [21].

the only serious adverse event (pneumonia) was considered to be due to the underlying disease. Adverse events were generally mild or moderate in severity, and treatment was not stopped for any PBI-4050-related adverse event. The most frequent adverse events were diarrhoea, nausea and headache. The PK profiles were similar among patients receiving PBI-4050 alone or in combination with nintedanib but were different in the combination with pirfenidone. The PK profile of PBI-4050 when added to pirfenidone showed reduced absorption and lower maximum observed plasma concentration of PBI-4050 and a faster metabolism (shorter half-life) compared with the other treatment groups, suggesting a drug-drug interaction between PBI-4050 and pirfenidone.

Decline in lung function as measured by FVC has long been used to monitor disease progression in IPF and has been identified as an independent predictor of mortality in IPF [22-25]. A reduction in the annual rate of decline in FVC \% pred, as determined by spirometry, is therefore a suitable primary endpoint for therapeutic studies in IPF. Current clinical practice guidelines recommend the use of one of the two available anti-fibrotic drugs, pirfenidone or nintedanib, for the treatment of IPF [1, 7]. Both drugs slowed the progression of lung function decline by approximately $50 \%$ as compared to placebo in large phase 3 clinical studies [26,27]. Although pirfenidone and nintedanib represent a significant improvement in the treatment of IPF, they do not represent a cure and both drugs have significant and sometimes intolerable side effects. Therefore, clinical research continues to focus on new therapies for the treatment of this progressive and often fatal disease.

FIGURE 2 Pharmacokinetic profile of PBI-4050 alone $(n=2)$ or in combination with nintedanib (NINT) $(n=4)$ or pirfenidone (PIRF) $(n=3)$ at week 12 (W12). Data are presented as mean \pm SD. HC: healthy controls. \#: these data are from a different study.

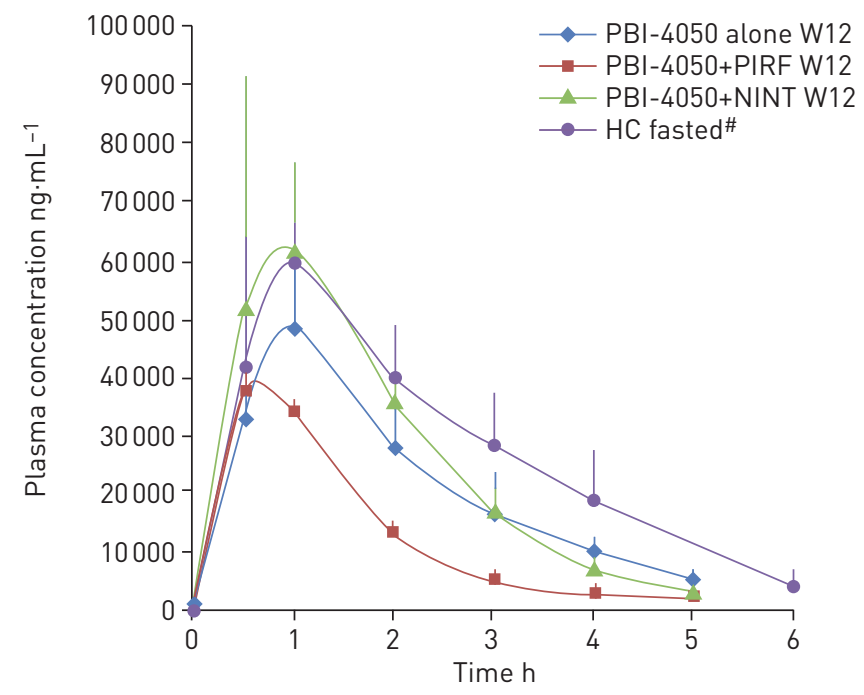


TABLE 3 Pulmonary function tests results

\begin{tabular}{|c|c|c|c|}
\hline Pulmonary function test & PBI-4050 alone & PBI-4050+nintedanib & PBI-4050+pirfenidone \\
\hline Patients n & 9 & 16 & 15 \\
\hline \multicolumn{4}{|l|}{ FVC $\%$ pred } \\
\hline \multicolumn{4}{|l|}{ Baseline } \\
\hline Mean \pm sD & $83.11 \pm 17.37$ & $71.31 \pm 17.05$ & $70.80 \pm 14.82$ \\
\hline Median & 79.0 & 69.0 & 68.0 \\
\hline Range & $58.0-109.0$ & $45.0-107.0$ & $45.0-100.0$ \\
\hline \multicolumn{4}{|l|}{ Week 12} \\
\hline Mean \pm SD & $82.00 \pm 16.57$ & $71.38 \pm 15.98$ & $68.11 \pm 15.79$ \\
\hline Median & 74.0 & 69.5 & 64.0 \\
\hline Range & $56.0-105.0$ & $51.0-98.0$ & $39.0-101.0$ \\
\hline \multicolumn{4}{|c|}{ Change from baseline at week 12} \\
\hline Mean \pm SD & $-1.11 \pm 4.46$ & $0.06 \pm 4.02$ & $-2.69 \pm 4.11$ \\
\hline Median & -1.0 & 1.0 & -4.0 \\
\hline $95 \% \mathrm{Cl}$ & $-4.5-2.3$ & $-2.1-2.2$ & $-5.00--0.4$ \\
\hline$p$-value $\#$ & 0.4759 & 0.9513 & 0.0240 \\
\hline \multicolumn{4}{|l|}{ FVC $\mathrm{mL}$} \\
\hline \multicolumn{4}{|l|}{ Baseline } \\
\hline Mean \pm SD & $2884 \pm 759.59$ & $2761 \pm 530.03$ & $2849 \pm 745.62$ \\
\hline Median & 2650 & 2735 & 2930 \\
\hline Range & $1750-4380$ & $1290-3480$ & $1580-4280$ \\
\hline \multicolumn{4}{|l|}{ Week 12} \\
\hline Mean \pm SD & $2872 \pm 811.82$ & $2763 \pm 520.70$ & $2747 \pm 813.67$ \\
\hline Median & 2770 & 2775 & 2720 \\
\hline Range & $1640-4330$ & $1430-3440$ & $1470-4360$ \\
\hline \multicolumn{4}{|c|}{ Change from baseline at week 12} \\
\hline Mean \pm SD & $-12.2 \pm 137.1$ & $1.9 \pm 127.6$ & $-102 \pm 137.8$ \\
\hline Median & -50.0 & 25.0 & -140 \\
\hline $95 \% \mathrm{Cl}$ & $-117.6-93.2$ & $-66.1-69.9$ & $-178.3--25.7$ \\
\hline$p$-value $\#$ & 0.7959 & 0.9539 & 0.0124 \\
\hline \multicolumn{4}{|l|}{ DLco \% pred } \\
\hline \multicolumn{4}{|l|}{ Baseline } \\
\hline Mean \pm SD & $45.22 \pm 11.62$ & $50.75 \pm 14.81$ & $49.07 \pm 16.33$ \\
\hline Median & 47.0 & 53.0 & 45.0 \\
\hline Range & $27.0-64.0$ & $24.0-70.0$ & $23.0-83.0$ \\
\hline \multicolumn{4}{|l|}{ Week 12} \\
\hline Mean \pm SD & $41.22 \pm 11.20$ & $49.25 \pm 14.03$ & $46.53 \pm 18.10$ \\
\hline Median & 41.0 & 49.5 & 45.0 \\
\hline Range & $26.0-56.0$ & $22.0-72.0$ & $18.0-83.0$ \\
\hline \multicolumn{4}{|c|}{ Change from baseline at week 12} \\
\hline Mean \pm SD & $-4.00 \pm 7.4$ & $-1.50 \pm 3.5$ & $-2.54 \pm 6.3$ \\
\hline Median & -3.0 & -3.0 & 0.0 \\
\hline $95 \% \mathrm{Cl}$ & $-9.7-1.7$ & $-3.4-0.37$ & $-6.0-0.9$ \\
\hline p-value & 0.1427 & 0.1073 & 0.1390 \\
\hline
\end{tabular}

FVC: forced vital capacity; \% pred: \% predicted; DLCO: diffusing capacity of the lung for carbon monoxide. ${ }^{\#}$ : p-value based on paired t-test.

A post hoc analysis of the three treatment groups showed no statistically significant changes in FVC (\% pred or $\mathrm{mL}$ ) after 12 weeks of treatment in patients receiving PBI-4050 alone or in combination with nintedanib $(\mathrm{p}>0.40)$. In contrast, a statistically significant decline in FVC was found during the same time period for patients in the PBI-4050+pirfenidone group (\% pred $\mathrm{p}=0.024 ; \mathrm{mL} \mathrm{p}=0.0124)$. This latter finding was not impacted by the disparity in baseline weight. Rather, the FVC findings are supported by the PK data, with a reduced absorption rate or lower maximum observed plasma concentration and a faster metabolism (shorter half-life) of PBI-4050 seen in the pirfenidone group.

Drug-drug interactions with pirfenidone have been reported in clinical IPF studies [28, 29], similar to the results observed in this PBI-4050 IPF study. Reduced efficacy of pirfenidone was observed in combination with $\mathrm{N}$-acetylcysteine in a 24-week randomised double-blind placebo-controlled study in IPF patients (PANORAMA study) [28]. This combination significantly reduced the adjusted rate of FVC decline compared to placebo and to pirfenidone $(125.6 \mathrm{~mL}$ over 6 months versus $34.3 \mathrm{~mL}$ over 6 months, 

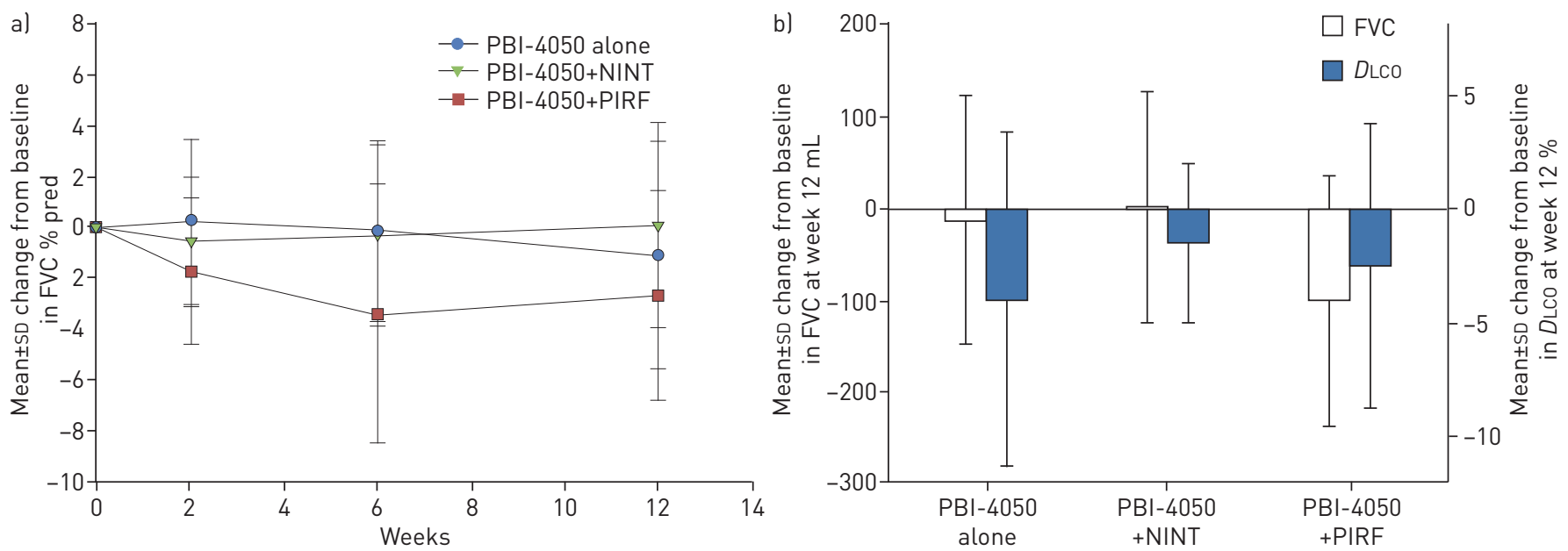

FIGURE 3 a) Absolute mean change from baseline in forced vital capacity (FVC) \% predicted following 12 weeks of treatment with PBI-4050 alone or in combination with either nintedanib (NINT) or pirfenidone (PIRF). b) Absolute mean change from baseline in FVC (mL) and diffusing capacity of the lung for carbon monoxide (DLCo) $(\%)$ following 12 weeks of treatment with PBI-4050 alone or in combination with either NINT or PIRF.

respectively; $\mathrm{p}=0.031$ ), with safety largely unchanged [28]. Reduced exposure was also observed with the combination of nintedanib and pirfenidone in a 28 -day randomised double-blind dose-escalation study in Japanese IPF patients [29]. Area under the concentration-time curve and maximum plasma concentration of nintedanib and its metabolites were reduced in patients receiving pirfenidone, but there were no changes in the PK profile for pirfenidone [29].

In summary, there were no safety concerns with PBI-4050 alone or in combination with nintedanib or pirfenidone after 12 weeks of treatment in patients with predominantly mild or moderate IPF. PK profiles for PBI-4050 alone and in combination with nintedanib were similar but were reduced with pirfenidone, suggesting a drug-drug interaction. FVC results were encouraging for PBI-4050 alone and in combination with nintedanib, despite limitations in sample size and study design.

Acknowledgements: Medical writing assistance, in the form of manuscript preparation and revision, was provided by Shalini Kanekar (Prometic Life Sciences Inc.) under the authors' conceptual direction and based on feedback from the authors.

Conflict of interest: N. Khalil has nothing to disclose. H. Manganas reports receiving grants from Prometic, during the conduct of the study. C.J. Ryerson reports clinical trial participation for Prometic, during the conduct of the study; and receiving grants and personal fees from Boehringer Ingelheim and Hoffmann-La Roche, outside the submitted work. S. Shapera reports receiving: personal fees for speaking engagements from AstraZeneca; grants and personal fees for participation in clinical trials as site PI, membership of advisory boards, grants for knowledge translation research, and honoraria for speaking engagements from Boehringer Ingelheim Canada; grants and personal fees for participation in clinical trials as site PI, membership of advisory boards, grants for clinical epidemiology research, and honoraria for speaking engagements from Hoffman-La Roche Canada, outside the submitted work. S. Shapera participated in clinical trials as site PI for Medimmune, Prometic Canada and Sanofi-Aventis, outside the submitted work. A.M. Cantin reports receiving grants and fees for consulting from Prometic, during the conduct of the study. P. Hernandez reports receiving: grants paid to his institution for conduct of research from Prometic, during the conduct of the study; personal fees for advisory board work from Actelion, personal fees for advisory board work and CME talks from AstraZeneca, grants paid to his institution for conduct of research and personal fees for advisory board work from Boehringer Ingelheim, grants paid to his institution for conduct of research from CSL Behring, Grifols and Cyclomedica, personal fees for advisory board work from GSK Novartis, Teva and Trudell, and grants and personal fees for advisory board work and CME talks from Bayer, outside the submitted work. E.E. Turcotte has nothing to disclose. J.M. Parker was employed by Prometic Life Sciences Inc., during the conduct of the study. J.E. Moran reports receiving personal fees, cash and equity considerations as an employee and shareholder of Prometic Life Sciences Inc., outside the submitted work. G.R. Albert is an employee of Prometic Life Sciences Inc. R. Sawtell was employed by Prometic Life Sciences Inc., during the conduct of the study. A. Hagerimana was employed by Prometic Life Sciences Inc., during the conduct of the study. P. Laurin reports receiving personal fees, cash and equity considerations as an employee and shareholder of Prometic Life Sciences Inc., outside the submitted work; and in addition, has a patent EP 2427417 issued, a patent EP 2427416 issued and a patent EP 2970088 pending. L. Gagnon reports receiving personal fees, cash and equity considerations as employee and shareholder of Prometic Life Sciences Inc., outside the submitted work; and has a patent EP 2427417 issued, a patent EP 2427416 issued and a patent EP 2970088 pending. F. Cesari reports receiving personal fees, cash and equity considerations as an employee and shareholder of Prometic Life Sciences Inc., outside the submitted work. M. Kolb reports receiving: grants from Prometic, during the conduct of the study; grants and personal fees from Roche, Boehringer Ingelheim and GSK, personal fees from Gilead and Genoa, and grants from Actelion, Respivert, Alkermes and Pharmaxis, outside the submitted work. 
Support statement: This study was funded by Prometic Life Sciences Inc. Funding information for this article has been deposited with the Crossref Funder Registry.

\section{References}

1 Raghu G, Collard HR, Egan JJ, et al. ATS/ERS/JRS/ALAT Committee on Idiopathic Pulmonary Fibrosis. An official ATS/ERS/JRS/ALAT statement: idiopathic pulmonary fibrosis: evidence-based guidelines for diagnosis and management. Am J Respir Crit Care Med 2011; 183: 788-824.

2 King TE Jr, Pardo A, Selman M. Idiopathic pulmonary fibrosis. Lancet 2011; 378: 1949-1961.

3 Hutchinson J, Fogarty A, Hubbard R, et al. Global incidence and mortality of idiopathic pulmonary fibrosis: a systematic review. Eur Respir J 2015; 46: 795-806.

4 Hopkins RB, Burke N, Fell C, et al. Epidemiology and survival of idiopathic pulmonary fibrosis from national data in Canada. Eur Respir J 2016; 48: 187-195.

5 Marshall DC, Salciccioli JD, Shea BS, et al. Trends in mortality from idiopathic pulmonary fibrosis in the European Union: an observational study of the WHO mortality database from 2001-2013. Eur Respir J 2018; 51: 1701603.

6 Ryerson CJ, Kolb M. The increasing mortality of idiopathic pulmonary fibrosis: fact or fallacy? Eur Respir J 2018; 51: 1702420 .

7 Raghu G, Rochwerg B, Zhang Y, et al. An official ATS/ERS/JRS/ALAT Clinical Practice Guideline: treatment of idiopathic pulmonary fibrosis. An Update of the 2011 Clinical Practice Guideline. Am J Respir Crit Care Med 2015; 192: e3-e19.

8 Raghu G. Idiopathic pulmonary fibrosis: lessons from clinical trials over the past 25 years. Eur Respir J 2017; 50: 1701209.

9 Selman M, King TE, Pardo A, et al. Idiopathic pulmonary fibrosis: prevailing and evolving hypotheses about its pathogenesis and implications for therapy. Ann Intern Med 2001; 134: 136-151.

10 Bellaye PS, Kolb M. Why do patients get idiopathic pulmonary fibrosis? Current concepts in the pathogenesis of pulmonary fibrosis. BMC Med 2015; 13: 176.

11 Plantier L, Renaud H, Respaud R, et al. Transcriptome of cultured lung fibroblasts in idiopathic pulmonary fibrosis: meta-analysis of publically available microarray datasets reveals repression of inflammation and immunity pathways. Int J Mol Sci 2016; 17: E2091.

12 Noble PW, Barkauskas CE, Jiang D. Pulmonary fibrosis: patterns and perpetrators. J Clin Invest 2012; 122 2756-2762.

13 Wuyts WA, Agostini C, Antoniou KM, et al. The pathogenesis of pulmonary fibrosis: a moving target. Eur Respir J 2013; 41: 1207-1218.

14 Gagnon L, Leduc M, Thibodeau JF, et al. A newly discovered antifibrotic pathway regulated by two fatty acid receptors: GPR40 and GPR84. Am J Pathol 2018; 188: 1132-1148.

15 Oh CK, Murray LA, Molfino NA. Smoking and idiopathic pulmonary fibrosis. Pulm Med 2012; 2012: 808260.

16 Schmid U, Liesenfeld KH, Fleury A, et al. Population pharmacokinetics of nintedanib, an inhibitor of tyrosine kinases, in patients with non-small cell lung cancer and idiopathic pulmonary fibrosis. Cancer Chemother Pharmacol 2018; 81: 89-101.

17 Genentech USA, Inc. Prescribing information for ESBRIET. 2017. www.gene.com/download/pdf/esbriet prescribing.pdf Date last accessed: May 29, 2017.

18 Lanthier J, Tanguay M, Larouche R, et al. Pharmacokinetics, safety and tolerability of PBI-4050, a novel anti-fibrotic drug, in healthy human subjects and in patients with stable renal impairment. Clin Pharmacol Drug Dev 2017a; 6: Suppl. 1, 3-72.

19 Miller MR, Hankinson J, Brusasco V, et al. Standardisation of spirometry. Eur Respir J 2005; 26: 319-338.

20 MacIntyre N, Crapo RO, Viegi G, et al. Standardisation of the single-breath determination of carbon monoxide uptake in the lung. Eur Respir J 2005; 26: 720-735.

21 International Conference on Harmonisation of Technical Requirements for Registration of Pharmaceuticals for Human Use (ICH). Medical Dictionary for Regulatory Activities, version 18.0. Geneva, ICH, 2015. www.meddra. org/sites/default/files/guidance/file/intguide_18_0_english.pdf Date last accessed: September 14, 2017.

22 Flaherty KR, Mumford JA, Murray S, et al. Prognostic implications of physiologic and radiographic changes in idiopathic interstitial pneumonia. Am J Respir Crit Care Med 2003; 168: 543-548.

23 Collard HR, Ryerson CJ, Corte TJ, et al. Acute exacerbations of idiopathic pulmonary fibrosis: an international working group report. Am J Respir Crit Care Med 2016; 194: 265-275.

24 du Bois RM, Weycker D, Albera C, et al. Forced vital capacity in patients with idiopathic pulmonary fibrosis: test properties and minimal clinically important difference. Am J Respir Crit Care Med 2011; 184: 1382-1389.

25 Zappala CJ, Latsi PI, Nicholson AG, et al. Marginal decline in forced vital capacity is associated with a poor outcome in idiopathic pulmonary fibrosis. Eur Respir J 2010; 35: 830-836.

26 Richeldi L, du Bois RM, Raghu G, et al. Efficacy and safety of nintedanib in idiopathic pulmonary fibrosis. $N$ Engl J Med 2014; 370: 2071-2082.

27 King TE, Bradford WZ, Castro-Bernardini S, et al. A phase 3 trial of pirfenidone in patients with idiopathic pulmonary fibrosis. N Engl J Med 2014; 370: 2083-2092.

28 Behr J, Bendstrup E, Crestani B, et al. Safety and tolerability of acetylcysteine and pirfenidone combination therapy in idiopathic pulmonary fibrosis: a randomised, double-blind, placebo-controlled, phase 2 trial. Lancet Respir Med 2016; 4: 445-453.

29 Ogura T, Taniguchi H, Azuma A, et al. Safety and pharmacokinetics of nintedanib and pirfenidone in idiopathic pulmonary fibrosis. Eur Respir J 2015; 45: 1382-1392. 